\title{
ART, LANGAGE ET EXPRESSION: RÉFLEXIONS SUR L'ART CONTEMPORAIN À PARTIR DES PENSÉES de MERLEAU-PONTY ET de FOUCAUlT
}

\author{
[ ART, LANGUAGE AND EXPRESSION: REFLEXIONS ON CONTEMPORARY ART FROM MERLEAU- \\ PONTY AND FOUCAULT ]
}

Salim Mokkadem*
Université de Montpellier, France

\begin{abstract}
Resumo: Et si les différences entre le ABstract: $E$ se as diferenças entre o matérialisme discursif de l'anarchéologue materialismo discursivo de Foucault, enquanto Foucault et la phénoménologie de l'entrelacs et anarquista, e da fenomenologia do tocar e da de la chair de Merleau-Ponty passaient par une carne, de MerleauPonty, concorressem para différence entre, d'une part, la conception du uma diferença entre, de um lado, a concepção sujet comme agent et processus éthopoḯtique de sujeito como agente e processo éticodes "vérités", pour le premier, et, d'autre part, poiético das "verdades ", para o primeiro, e, por par une autre définition de l'art qui ne serait pas outro lado, para outra definição de arte que não expression du sujet ou style perceptif/expressif, seria expressão de um sujeito ou estilo comme pour Merleau-Ponty, mais, perceptivo / expressivo, como para Merleaulittéralement, qui serait effraction dans le Ponty, mas, literalmente, que seria trangressao discours du monde d'un langage aux limites de na fala do mundo de uma linguagem nos limites tout énoncé dicible, pensable, énonçable dans de todo enunciado proferível, pensável e l'épistémè où il apparait justement comme hors enunciavel na ciencia, onde aparece limites? C'est la thèse que nous développons en precisamente como fora dos limites? Esta é a montrant que la figuration et le visible pour tese de que tratamos, neste artigo, visando Merleau-Ponty demeurent la chair du monde, mostrar que figuracaao e o visível para dans une anthropologie proche d'une relecture Merleau-Ponty permanecem encarnadas no néoplotinienne du monde, et que l'informe, mundo atraves de uma antropologia proxima de l'hétérotopie, le rapport au temps et au non uma releitura neoplotiniana do mundo, e que figuratif (sortie du monde classique) permettent informa, a heterotopia, a relacao ao tempo e ao à Foucault de penser l'oeuvre d'art comme não-figurativo (deixando o mundo clássico) absence de folie ou comme sortie réelle de son permitem a Foucault pensar a obra de arte époque par la production d'une singularité qui como ausência de loucura ou como saída real échappe autant à la figuration et à la de seu tempo pela produção de uma représentation de l'épistémè où elle apparait singularidade que escapa tanto à figuração e à qu'aux apories de la présentation de l'autre dans representação da ciencia na qual aparece quanto le même. C'est dans l'esthétique et le lien à nas aporias da presentificação do outro no l'aisthésis qu'il faudrait alors comprendre à la mesmo. É na estética e na ligação com a fois leur rapport à Hegel et à Platon, et leur sensibilidade que devemos, pois, entender conception de la vie humaine. Le rapport à "la tanto a relação deles com Hegel e Platão, vérité" suppose, dans les deux pratiques, un quanto a concepção deles da vida humana. A certain rapport à "la" liberté qui est, de façon relação com a "verdade" supõe, em ambas as tourmentée et tragique, un certain rapport à soi, práticas, uma certa relação com a "liberdade"
\end{abstract}

* Doctorat en Philosophie. Professeur de philosophie à la Faculté d'Éducation de l'Université de Montpellier,EA3749,Lirdef.m@ilto: salim.mokaddem@gmail.com 
aux autres et au monde.

Mots-CLÉs: Parrhésia; éthopoiétique; qué, de mañ énoncés et discours; sensible et intelligible; com o mundo.

sujet et subjectivation

Palavras-chave: Paresia; ético-poiético; enunciados e discurso; sensível e intelível; sejeito e subjetividade

Werleau-Ponty dans la Phénoménologie de la perception (PP, 1945) et 1 surtout dans les travaux sur l'entrelacs de la chair (Le visible et l'invisible, VI, 1960) pose la question du langage de la perception et de la signification du sens dans les signes et dans les expressions. Sa thèse porte l'idée que tout est langage (Signes, 1960) et que le langage est la matrice du sens et du non-sens (Sens et non-sens, 1948).

L'expression renvoie à l'intuition perceptive du sens et à une signification ambiante, mondaine (Umwelt) qui elle-même repose la question du rapport du corps, dans la fonction signifiante, d'une part, et, d'autre part, de l'histoire des liens entre la pensée et le corps (lectures de Malebranche, Spinoza et Bergson faites alors en Sorbonne par Merleau-Ponty, et cours édités chez Vrin par J. Deprun). On peut donc parler d'une phénoménologie esthétique ou esthésique (Petrucia Nobrega, 2010) du signe et d'une structure matérielle du signe pris dans la chair du monde (La structure du comportement, 1942)).

La nature est donc tout à la fois un langage et possède, de façon qu'il faudra préciser et détailler, un sens anté-prédicatif - ce qui pose la question de la rationalisation possible du monde dans l'usage de ce type de syntagme - et qu'il faut toujours comprendre comme étant déjà pris et prérequis dans une culture donnée : l'humanité est prise dans ce couple culture-nature dont il est difficile de sortir, pour la simple et bonne raison qu'il est aussi instituant et institué dans l'histoire de la pensée, et parce que l'humanité est l'expression parlante de cette insertion de la culture dans la nature, ou, si l'on veut, parce que le signifiant est premier pour l'humanité jamais nue car l'ordre du langage encode et formalise le lien de la pulsion au désir par un nouage anthropologique qui confère au discours une réalité ontologique et structurel dont il est difficile, voir impossible de se libérer (Lacan, Ecrits, 1966).

L'autre de soi, n'est pas un autre que soi ou un autre du soi, ou un autre de soi : il est l'intentionnalité signifiante appelant une lecture du monde à partir de lui et donc en sortant de soi (ek-stase) pour aller vers l'autre et faire sens avec et par lui dans une expérience vécue (Erlebnis). Tout sens est donc ou devient de ce fait politique car il relie la nécessité d'aller à l'autre en passant par une ek-stase, une sortie de soi, pour aller dans le monde de l'autre, dans l'être avec l'autre, le Mitt-sein thématisé par Heidegger (Sein und Zeit, 1927). Il y a basculement du mondain de l'Umwelt vers l'être avec l'autre, jusques et y compris dans la haine et l'affect sans visage (ce qui est signifié par le sens éthique de l'engagement et le risque de la perte dans l'affect de haine dans la fin de « La guerre a eu lieu »).

On ne peut donc séparer : esthétique et linguistique (au sens structural) et éthique et politique (au sens existentialiste) dans la mesure où je suis à l'autre comme il est à moi dans la dialectique du sens et de son remplissage par sa présence ou son absence dialectique qui noue sensible et intelligible dans son être-là. Ceci est manifeste dans la sexualité qui ne peut avoir lieu et sens en dehors de la reproduction et qui pourtant se détache de la pulsion, pour l'être humain, afin de construire du sens des émotions, une histoire, faite de désirs et d'engagements moraux et affectifs. Le sexe, pour nous, est à jamais corrélé à l'histoire de notre éducation, subjectivité, dispositif éthique, qui oblige à prendre en compte l'altérité humaine, corporelle, émotionnelle, 
pathique, dans le temps et dans l'espace. Freud montre bien, par ailleurs, que toute pulsion s'historicise dans un corps et dans des zones érogènes qui sont elles-mêmes des montages entre nature biologique, pulsion physiologique et expression culturelle signifiante; et la politique des rencontres sexuelles ne se fait jamais dans l'absence de la référence à ce qui l'autorise ou lui confère ses légitimités et ses logiques idiosyncrasiques.

Deux questions viennent alors se poser légitimement :

- pourquoi Merleau-Ponty, pour parler du sensible, convoque la référence épistémologique et phénoménologique, à Husserl, celui des Leçons sur le temps et des Recherches logiques, plutôt que le recours à l'art ou à l'artiste lui-même ? Ce sera alors le sens et la pertinence de la logique de mobilisation de toutes les interrogations sur le cinéma, sur Cézanne, sur la figuration de l'infigurable en arts plastiques, et les mobilisations de la nouvelle psychologie et du cinéma pour penser le sensible, qui seront alors à interroger quant à sa pertinence.

- pourquoi la figure du corps singulier demeure encore le référent pour penser la pertinence de l'action politique de Merleau-Ponty, jusques et y compris dans Humanisme et terreur ou Les aventures de la dialectique (respectivement édités en 1947 et en 1955), et non pas le corps social, le parti, le peuple, l'histoire, l'intensité de l'action ou de la pulsion (Deleuze et Guattari, reprenant Nietzsche et Spinoza, à leur façon, feront cette psychanalyse politique dans Anti-Oedipe) ? Pourquoi l'artiste donnera-til plus la vérité du monde, de l'histoire, du sujet engagé dans l'existence que le parti politique, l'idéologie et l'adhésion collective à un mouvement porté par le sens de l'histoire?

On rappelle que ce sont justement les totalitarismes rouges et bruns qui mèneront Merleau-Ponty à quitter les idées politiques esthétiques de Sartre (1947), du Marx engagé dans la dictature du prolétariat, et à récuser, in fine, toute adhésion à un communisme d'Etat, celui du Parti, pour déboucher sur une aporie éthique qui est celle d'un humanisme ouvert et en recherche de lui-même. Car, le paradoxe et la contradiction tragique est que c'est justement cet humanisme déclaré, formel, qui a aussi produit le même universalisme abstrait, le même désir du bien en même temps qu'il instituait, pour se réaliser dans le moment de son affirmation, son contraire. L'humanisme produit la terreur de l'anti-humanisme au nom de l'humanisme quand il fait une hiérarchisation entre les civilisations, les valeurs, les cultures et les définitions de la vérité de l'humanité ; son autre ou son contraire absolu seront alors des ennemies ou des anti-valeurs (l'esclavagisme, le nazisme, les totalitarismes, se faisant toujours au nom de l'Universel et de la Justice des droits de l'homme).

Merleau-Ponty parle alors d'un infra-rationnel, d'une nature qui ne se résorbe pas toute dans la culture ou dans la raison : au contraire de Hegel qui en fait, à la façon d'Aristote, un impensable, dans sa théorie encyclopédique qui fait que la Nature est une Logique de la Nature comme Logique de l'être, le phénoménologue pose qu'elle est impensable de fait et non pas de science. Il stipule qu'il nous faut une ontologie sauvage pour penser l'entrelacs du corps dans la nature et dans le désir (la chair est ce concept de l'obscur vincula physica), mais il pose également que cette nature n'échappe pas à la saisie, par le logos ou l'action de l'artiste, à une compréhension ouverte du sens de l'immédiat de son donné ; paradoxalement, le donné de cette immédiateté passe alors par le travail continue et patient de l'artiste qui rend, par son 
œuvre et son travail, au monde perceptif, le caractère presque transcendantal de son apparition sauvage et insaisissable par le concept formel.

Qu'est-ce que l'expression artistique enseigne au philosophe, comme au sens commun, de vraiment différent, par son travail poïétique, sur la nature du monde ? En quoi la phénoménologie du corps engagé dans la chair du monde autorise-t-elle une autre vision du visible et un autre savoir du savoir que celui des savants et des philosophes? Dans la mesure où le travail de l'œuvre artistique reconduit le monde originaire sur lequel elle se fonde et dans laquelle elle s'exprime pour lui donner sa vérité primaire et originaire.

$\mathrm{Si}$ la science de la sensation échappe à la rationalisation de type physicomathématique et formelle, au sens des Wissenchaffenlehre, quel est donc son type de savoir, ses modalités épistémologiques propres, pour que la vérité du monde de la sensation soit connaissable, sue, intuitionnée ? Il y a une véritable impasse du recours à la métaphore et à la métonymie du concept phénoménologique qui décrit, suspend, analyse, recompose et intuitionne le monde mais ne peut pas agir par des schèmes ou des catégories sur lui pour l'appréhender comme tel. L'impasse de la philosophie critique de Kant vient qu'elle s'est trop vite séparée de l'être pour penser les conditions du phénomène sans en faire la réduction à même le lieu de son apparaître. C'est le thème transcendantal lui-même qui est interrogé : peut-on par ce biais éviter l'idéalisme inhérent à la mise entre parenthèse du monde par un sujet qui redupliquerait, du fait de l'acte noético-noématique, les coupures formelles entre objet-sujet, matière-forme, monde-ego transcendantal, transcendance et visée, conscience et phénomène ?

L'action est elle-même un langage qui, pour être saisie comme telle, suppose une inscription et une situation dans le monde (Être et néant, 1942) obligeant l'individu, en pleine conscience, à vouloir ses choix. Or, l'hypothèse du corps s'échappant à lui-même (inconscient freudien, ou entrelacs de la chair du monde dans mon corps perceptif) fait que le monde m'échappe, et que je ne puis donc le comprendre qu'en en excluant la signification expressive totale qui manque structurellement et ontologiquement à ma visée toujours située hic et nunc dans le présent de ma perception.

Percevoir, c'est manquer l'être du monde et atteindre les limites de mon corps.

Pourquoi alors Merleau-Ponty ne pense-t-il pas ces expériences limites comme étant des états de corps permettant la saisie de l'être, justement, dans ses effractions multiples (jouissances, excès, angoisse, etc.), comme Bataille le fait dans La Part maudite ou L'Erotisme, par exemple ? Quelles sont les limites de la philosophie esthétique de la critique esthétique de la phénoménologie ? S'agit-il alors de rejouer les antinomies du Corps apollinien, rationnel contre le Corps dionysiaque ? Au risque de recommencer et de répéter les errances de l'épistémé naturaliste et organisationnelle des substantialisations de l'âge classique et de confondre, par le recours à la dualité FormeMatière ou Idée et genesis, l'objet avec la catégorie qui le désigne ?

$\mathrm{Ou}$ bien est-ce parce que Merleau-Ponty est encore pris dans un ordre du discours, un rapport au langage, un Usage du discours plein, transparent, celui du sujet cartésien, propre à une certaine philosophie de la représentation (Foucault, Les mots et les choses, 1966) qu'il ne peut sortir du thème transcendantale du sujet husserlien des Méditations cartésiennes ? Ou bien, le philosophe manifeste-t-il en creux l'impossibilité de dire l'être obscur qui traverse le corps du philosophe, corps pris par la guerre et les contraintes morales et politiques qu'elle impose à tout un chacun et qui fondent autant d'attitudes éthiques, politiques, morales, relevant d'analyses phénoménologiques spécifiques, comme celles-ci : qu'est-ce que la résistance, la collaboration, la passivité, l'attentisme, la colère, la révolte, l'indifférence, le malaise de la contradiction vécue empiriquement et non plus discursivement, impuissance, ou hérö̈sme suicidaire ou lâcheté résignée, en période de guerre ? Peut-on faire l'épochè de la guerre comme crise totale du sens et de la liberté ? Sartre le pensait ; d'où son existentialisme cartésien et 
son anti-hégélianisme se manifestant dans son refus de penser l'inconscient freudien comme une logique de l'être et du sens. Merleau-Ponty, plus conséquent, admet qu'il existe de l'altérité ontologique et herméneutique dans l'histoire du sens et des vérités produites par la raison dans l'histoire de l'humanité.

On peut, si l'on veut comprendre cette position qui consiste à tenir sur le sujet (et non pas la subjectivité) contre et dans l'histoire, analyser la façon dont MerleauPonty conçoit l'art et les finalités de l'art moderne (post-industriel), par exemple, la peinture de Cézanne ou le cinéma de la nouvelle vague, et comparer alors ses analyses esthétiques à la façon dont Michel Foucault pense l'art visuel (Magritte ou Manet) et la littérature. On remarquera, sans en tirer des conséquences ad hoc, que Foucault est d'abord plus sensible à la musique, aux arts temporels qu'aux arts de l'espace, cherchant dans ceux-là une façon de sentir le temps et l'espace comme une possible sortie du monde, et dans ceux-ci, les arts de l'espace, plutôt le lieu d'une hétérotopie ou d'une politique de la subversion ou des limites de l'assignation ontologique à la présence et à l'être-là. Là où Merleau-Ponty recherche un nouvel humanisme pour son temps ou les raisons et les concepts pour refonder un humanisme qui ne soit pas la cause d'une terreur possible (le schéma hégélien de la Terreur comme folie de l'abstraction vide préfigurant ainsi la logique des purges staliniennes ou les totalitarismes de l'Idée pure séparée du sensible), Foucault cite plutôt Hölderlin, Nerval, Artaud, Bataille, pour installer la folie au cœur de la raison et du jeu des vérités dont elle est la garde et la prétendue connaissance. Foucault voit dans la folie de ces artistes non pas une coquetterie esthétique ou une posture d'avant-gardiste excentrique, mais la nécessité de faire exploser les normes du bon goût et des limites de la rationalité du moment du fait que ce que dit l'artiste excède les données sensibles et immédiates de ce que donnent à penser les systèmes des vérités et des savoirs de son temps. MerleauPonty est plus proche de Descartes, par la tradition kantienne et le thème transcendantal du sujet, que Foucault, plus en prise sur le désir et la pulsion, au sens de Nietzsche et de Freud.

L'art pour Foucault est en effet plus explosif, transgressif, aux limites de la forme acceptée de son expression, et en cela plus proche de la folie et d'une expérience des limites que d'un accès réglé et apollinien à l'être : le dérèglement des sens et des discours, des pratiques et des vies, produit l'obligation de transcender la vérité des pouvoirs qui les contrôlent et de mettre au grand jour la part maudite de l'expression artistique. Car elle ne vise pas à chercher ou à produire une bonne forme ou une esthétique, au sens néo-kantien du terme, mais à excéder les limites territorialisées des assignations normées au sens, à la rationalité contrôlée des connaissances produites par des logiques de pouvoir et des dispositifs techniques visant à contrôler les forces des sujets et à cadrer leurs effets dans une optique d'utilisation de leurs énergies et de leurs productions. Pour Foucault, l'artiste expérimente par un devenir soi, un souci de soi, une façon d'être au monde qui le rend inacceptable pour le monde autant que pour luimême. Il s'agit là aussi de se déprendre de soi, de penser et de faire autrement qu'on n fait pour oser une autre vie possible.

C'est pour quoi l'expérience des limites traduit pour Michel Foucault le fait de produire au jour, et souvent de manière scandaleuse, les raisons qui fondent la logique d'une rationalisation des manières de sentir, de percevoir, de juger les objets du monde, ou d'aller aux limites des savoirs produits par des énoncés, des questions, des problèmes, des connaissances, des interrogations, des violences, des recherches de vérité qui se présentent comme évidences pour notre présent. Ce travail archéologique recherche des domaines de légitimité des vérités et histoire des raisons des discours sur les objets, les champs, le enjeux, les intérêts de ces domaines - passe par une quête des éléments, des frontières, des passages de frontières, qui délimitent des champs épistémologiques distincts, consacrés historiquement, et ininterrogés dans leur 
pertinence actuelle. Double travail que l'art, sans auteur ni sujet assigné autre que celui à venir dans le moment même où il déconstruit les évidences du monde (ce qui l'apparente au philosophe dans sa suspension du monde et de son sens), introduit dans le jeu ordonné de la forme et du contenu en bougeant les lignes et les codes de leur convenance technologiquement institué, par tout un système académique de rituels d'acceptation, de reconnaissance, de validation leur conférant le statut d'œuvre artistique.

Il convient plutôt, selon Foucault, d'en faire (ou d'en défaire ou d'en refaire) la généalogie stricte afin d'interroger l'identité de nos épistémaï, c'est-à-dire, d'objectiver, d'extérioriser, de mettre au jour et à jour, la validité de nos connaissances assurées bien naïvement de leur « scientificité » et de leur « vérité ». L'art, comme champ d'objets et de pratiques, n'échappe pas à ce mouvement consistant à interroger ce qui le désigne comme tel. Il est le fruit d'une politique des vérités et n'a pas l'innocence qu'on lui prête trop facilement.

Pour Merleau-Ponty, l'art est la vérité du sensible et du visible en ce qu'il expose, par la vérité expressive de son langage propre, les voies manifestes et les voix silencieuses et clamantes du réel qui structure la perception du sensible et tout monde possible. On peut ainsi dire que Cézanne rend visible le Mont Ventoux et, comme le dira Deleuze, que les pommes de Cézanne sont plus des seins de femmes et donc renverse notre regard pour nous montrer le devenir des choses dans leur possible. Il y a un leibnizianisme dans cette attitude de réversibilité harmonieuse, ou que rend harmonieuse, l'attitude du philosophe regardant avec son esprit et pensant avec son œil le monde rendu visible par cette transformation réversible du peintre. Merleau-Ponty nous rend le monde plein de signes, parlant et bruissant de langages indirects et inachevés (comme l'est sa philosophie selon Paul Ricoeur) en tant qu'il fait de l'œuvre d'art, au fond, la continuité de l'activité spéculative du philosophe.

Pour Michel Foucault, l'art, et spécialement, la littérature, est plutôt la poursuite d'une tentative de sortie du monde, dans le monde de l'existence rendue impossible, du fait même des conditions du langage (« Ceci n'est pas une pipe », éd. Fata Morgana), des normes esthétiques de l'art, et de la possibilité de produire, d'une façon inattendue, une proposition affirmative négative (comme le sont les mots de Bataille) excédant le langage donné, non pas en l'esquivant, ou en passant en dehors, ou au-dessus, ou en dessous du langage, mais, bien plutôt, en s'installant en lui, dans son impossibilité à ne pas dire ce qui le limite et qui oblige l'écrivain (Faulkner, Genêt, Roussel, par exemple) à une esthétique de la transgression qui conserve donc, comme chez Sade, l'ordre du langage, mais pour mieux en affirmer l'absurdité ou l'irrationnel de sa rationalité affirmée. Ainsi de l'œuvre de Maurice Blanchot ou de René Char, reprenant la voie dangereuse ouverte par la transgression de Nietzsche ou le nihilisme post-moderne. Une affirmation négative est une proposition qui, en disant ce qu'elle dit, exclue ce qu'elle n'autorise pas à dire ou à énoncer. En ce sens, tout art est un bouleversement authentique des lois du langage et la tentative d'une expression totale et sans appel, paradoxalement, sans formes assignables autres que l'irruption de son événement singulier comme jaillissement inédit et pourtant rendu possible par ce qui le nie ou le limite.

Il n'y a pas d'universel du langage qui ne soit confronté à ses limites singulières et à ses vérités historiques. La diagonalisation de l'histoire par la politique, et inversement, oblige l'artiste à être littéralement hors normes ou à dénormaliser les catégories et les logiques de la représentation et de l'action. La littérature, comme expression de ce mouvement, est cette aventure de dépossession dans la contradiction incessante puisqu'elle doit mener cette opération subversive dans le champ du langage qu'elle bouleverse de façon dangereuse et insensée. La forme du langage de l'œuvre traduit les fins de l'artiste, traditionnellement pensé comme sujet, ou cause de soi et 
cause de son œuvre ; c'est l'œuvre qui produit l'artiste et la littérature met le monde dans l'obligation d'avoir à parler autrement qu'il ne parle du langage du fait même de la fracture dans l'événement du langage que produit l'irruption éthopoïétique du discours aux limites, tenu à l'intérieur du monde, dans son ordre officiel et contre ses vérités immuables, silencieuses et normées par des systèmes esthétiques redoublant des classifications, des découpes, des logiques identitaires et figées dans le refus de penser, sentir, parler, ailleurs que dans les codes institués par le bon goût et les savoirs du jour.

C'est pourquoi la folie en dit plus long sur la prétendue santé qui la contrôle, la prison sur les rigueurs et les logiques punitives de la loi, la maladie sur la vérité de la médecine, et la sexualité du souci de soi et des aphrodisia sur les normes et les logiques du pathologique au cœur des raisons éthiques des hédonismes contemporains. En fait, les expériences limites et l'éthique de l'existence, l'éthopoïétique, permettent à Foucault de se passer d'un recours à l'art comme discipline pour penser et l'éthique et l'existence. Dans les différents volumes de l'Histoire de la sexualité $(1976,1984,2018)$ Foucault montre que le travail de subjectivation, d'ascèse, de discipline et de souci de soi, tiennent lieu de pratique éthique, poiétique, esthétique, politique, pédagogique, philosophique dans la mesure où ces différentes sagesses, pratiques, ascèses, transforment, de l'intérieur et à l'extérieur de lui-même, le sujet qui pense et agit selon une parrhèsia son rapport à soi, aux autres, et au monde.

Foucault pense alors l'art comme l'autre de la culture et son autre à l'intérieur des limites négatives de ses exclusions, de ses lois, de ses normes, de ses vérités. L'art n'est pas un domaine séparé de la vie, du vivant, de l'existence quotidienne et il n'a pas à revendiquer pour lui un régime spéciale ou un droit d'exception pour asseoir des privilèges ou des prérogatives de puissance signifiante car il est effraction dans la vie d'une vie plus vivante que ce qui produit l'œuvre, l'artiste, le musée et ses institutions. L'art se subsume en vie vécue et en autopoḯse ; il devient alors, art ou technique de vie (technè tou biou) dans une éthopoiétique constante, travail permanent de soi sur soi, où le sujet a à se faire, en se déprenant de lui-même, par un souci constant de son être profond, de sa vérité et de ses pratiques dans un contexte où tout est l'objet d'un travail continu et discret sur lui-même, les autres et le monde qui l'entoure, le précède et qu'il veut faire advenir par cette application minutieuse et rigoureuse de soi sur soi, avec les autres, pour et par eux, dans un monde qui ne reconnaît encore que les taxinomies des inclusions et des exclusions, et les biopolitiques d'arraisonnement du sensible par des vérités ordonnées et limitées par une histoire des savoirs et des pouvoirs. On peut dire que Foucault donne à l'art, et aussi à la philosophie qui en fait l'analyse spécifique, la mission de contredire les vérités que Merleau-Ponty veut, a contrario, confier au monde sauvage des artistes et au regard de l'esprit réconciliateur du philosophe. Foucault assigne à l'artiste, entre autre, comme effet non recherché de ses puissances, une volonté de vivre la vie autrement qu'on ne vit et de changer le monde de manière hétérotopique quand Merleau-Ponty, de manière platonico-hégélienne, demande à l'artiste de reconnaître la vérité et la logique de l'esprit qui réconcilie la chair du monde et les aveux de son entrelacs.

Pour ne pas conclure:

Pour Merleau-Ponty, l'art demeure sotériologique, voir eschatologique, dans la mesure, où, comme chez Platon, le philosophe se transforme en artiste pour penser avec sagesse et avec application constante son rapport à la vérité, au monde, et à l'histoire. Il s'agit pour lui de conduire sa vie du mieux possible afin de ne pas déroger aux lois essentielles de la philosophie (Eloge de la philosophie). Il y a chez Merleau-Ponty un rapport à l'essence ou au mode sub specie aeternitatis, qui n'existe plus du tout chez Michel Foucault qui entretient un rapport archéologique ou anarchéologique à l'histoire, où l'histoire est celle des libertés et des contraintes dans le même temps où elle se défait par le jeu de sa facture, plus ou moins déterminée. Les deux philosophes 
voient dans l'art une façon de se libérer du monde et de ses certitudes ; mais la subjectivation foucaldienne n'est pas la contemplation phénoménologique du chiasme entre soi et la chair du monde. Elle est plus tragique, plus solitaire, plus indécise sur les fins et les valeurs qu'elle porte. Au fond, l'antiplatonisme de Nietzsche, que Foucault reprend à son compte, rend encore plus nominaliste la position de Foucault sur l'art contemporain : il n'a rien à nous offrir que de chercher à vivre l'immanence de notre singularité historique.

Être libre pour Foucault suppose donc d'avoir à se libérer constamment de ce qui favorise, dans l'histoire, le retour du Même et empêche l'invention de soi ; chez Merleau-Ponty, être libre suppose une révélation ou une conversion du regard vers un essentiel que la phénoménologie situe dans un rapport à la réalité qui passe par la reconnaissance des continuités labiles des langages du sensible et du perceptif, du sens et du non sens. Il y a chez Merleau-Ponty un secret du monde, une Idée des choses, et une vérité de leur interprétation, là où Foucault voit plutôt dans l'être au monde un jeu obscur de forces et de contraintes dont le philosophe a à se libérer par une déprise constante de soi, aux limites d'une expérience tragique.

Il nous faut bien reconnaître que la raison et le réel ne correspondent pas toujours et essentiellement aux vœux de l'Idée du Beau, du Bien et du Vrai.

\section{RÉFERÉNCES}

Heidegger, Martin, Etre et temps (1927) : Authentica, trad. Martineau, 1986

Bataille, Georges, La Part maudite : Minuit, Paris, 1949

Bataille, Georges, L'Erotisme : Minuit, Paris, 1957.

Clero, Jean-Pierre, " Maurice Merleau-Ponty et la guerre » : in Revue philosophique de la France et de l'étranger, 2002/3 (tome 127), pp. 315-331.

Foucault, Michel, Les mots et les choses : Paris, Gallimard, 1966.

Foucault, Michel, «La pensée du dehors » : Paris, Minuit, revue Critique n²29, juin 1966.

Foucault, Michel, Ceci n'est pas une pipe : Fontfroide-le-Haut, éd. Fata Morgana, 1973.

Foucault, Michel, L'ordre du discours : éd. Gallimard, Paris, 1976.

Foucault, Michel, HS, II, Le souci de soi : Gallimard, 1986.

Foucault, Michel, La sexualité (1964) : éd. Hautes études/Gallimard/Seuil, Paris, 2018.

Foucault, Michel, HS, IV, Les aveux de la chair : Gallimard, 2018.

Lacan, Jacques, Ecrits, 1966 : Paris, Seuil.

Merleau-Ponty, Maurice, Phénoménologie de la perception : Gallimard, 1945.

Merleau-Ponty, Maurice, La structures du comportement : PUF, 1942.

Merleau-Ponty, Maurice, Sens et non-sens : Nagel, Paris, 1948 .

Merleau-Ponty, Maurice, Eloge de la philosophie: Gallimard, 1953.

Merleau-Ponty, Maurice, Signes: Gallimard, 1960.

Merleau-Ponty, Maurice, Le visible et l'Invisible, Gallimard, 1960.

Mokaddem, Salim, « De Husserl à Freud. Un procès sans sujet » in Jean-Claude Beaune, dir., Phénoménologie, psychanalyse : étranges relations : éd. Champvallon, Seyssel, 1996.

Mokaddem, Salim, "Bataille dans la philosophie » in L'héritage impossible : éd. PUL, Montpellier, 1997.

Mokaddem, Salim, Foucault. Une vie philosophique : éd. Champ social, Nîmes, 2014.

Nobrega, Petrucia, "Le corps comme œuvre d'art : notes sur une ontologie sensible chez Merleau-Ponty » in Le corps en acte : centenaire Merleau-Ponty, Collège de France : éd. PUN, Nancy, 2010, pp. 273-285.

Sartre, Jean-Paul, Situations 1. Février 1938-septembre 1944 (1947) : Gallimard, 2010. 